\title{
GALAXIES GIVING RISE TO MGII ABSORPTION SYSTEMS IN QUASAR SPECTRA
}

\author{
Jacqueline Bergeron \\ Institut d'Astrophysique CNRS \\ 98 bis, bd Arago \\ 75014 PARIS, FRANCE
}

\begin{abstract}
The properties of the MgII absorption line systems detected in quasar spectra are briefly summarized. If these absorption systems arise in intervening galaxies, one can estimate their characteristic size and luminosity, and predict their identification rate. Results of a search for the absorbers is presented for a sample of $13 \mathrm{MgII}$ systems at $0.16 \leq \mathrm{z} \leq 0.79$. Strong constraints on the luminosity function are derived from the high actual identification rate and observed galaxy luminosities. Other properties of these absorbing galaxies are discussed, especially their spectral type and spatial environment.
\end{abstract}

\section{INTRODUCTION}

The early suggestion of Wagoner (1967) and Bahcall and Spitzer (1969) that the absorption line systems may arise in intervening galaxies is now confirmed at least for the sharp metal-rich absorption line systems at redshift $\mathrm{z}<\mathbf{1}$.

This talk will concentrate on the properties of these absorbers and of their parent galaxies. Sargent (1987) in a previous IAU symposium gave an overview of the properties of other types of systmes and of their applications in cosmology.

The intervening origin of metal-rich systems has been established by statistical arguments and by the identification of the absorbing galaxy. Young et al. (1982) have shown that the distribution of the number of CIV absorption redshift per line of sight is Poissonian. These large unbiased CIV samples also provide some insight on the interstellar gas physical properties in high redshift galaxies, 
such as spatial extent, ionization level, density and heavy element content. An alternative approach is to search for CaII absorption from the outer regions of local galaxies. The first attempt was made by Boksenberg and Sargent (1978) for the galaxy-quasar pair NGC3067-3C232 with detection of CaII absorption at a projected radial distance of $16.6 \mathrm{kpc}$, assuming a value for the Hubble constant $\mathrm{H}_{o}$ of $50 \mathrm{~km} \mathrm{~s}^{-1} \mathrm{Mpc}^{-1}$.

The most direct approach is to identify the galaxy responsible for typical MgII or CIV absorption systems. Since available CIV samples concern only high redshift $(z>1)$ systems, searches for the absorbing galaxies have only been attempted for MgII systems. A successful identification was recently obtained for the $\mathrm{z}=0.430 \mathrm{MgII}$ system in PKS2128-12 and the projected separation of this pair is $64 \mathrm{kpc}$ at the galaxy redshift (Bergeron 1986).

We first give a summary of some statistical properties of metal-rich absorption line systems and discuss possible cosmological evolution of their density per unit redshift. We briefly present the different phases of ionization level found for higher $z$ absorbers. We then describe results of our search for galaxies giving rise to $\mathrm{MgII}$ systems, and compare the statistical properties of the absorbing galaxies inferred from MgII absorption line samples with those obtained from their identification.

\section{AVERAGE SIZE OF THE ABSORBERS AND COSMOLOGICAL EVOLUTION}

For Friedmann models with zero cosmological constant and a decceleration parameter $\mathrm{q}_{o}$, the number of absorption line systems per unit redshift is given by

$$
\frac{d N}{d \mathrm{z}}=\frac{c}{H_{n}} n_{o} \sigma_{o}(1+\mathrm{z})^{1+\alpha}\left(1+2 \mathrm{q}_{o} \mathrm{z}\right)^{-0.5}
$$

where $n_{o}$ and $\sigma_{o}$ are the density and projected cross-section on the sky of the absorbers at $\mathrm{z}=0, c$ is the speed of light and $\alpha$ represents a possible cosmological evolution.

The $n_{o} \sigma_{o}$ product is estimated using a Schechter luminosity function and a Holmberg radius-luminosity scaling law

$$
\begin{aligned}
& d n=\phi_{o} x^{-5 / 4} e^{-x} d x, \\
& \sigma=\sigma_{*} x^{5 / 6},
\end{aligned}
$$

with $x=L / L_{*}, \phi_{o}=310^{-3} \mathrm{Mpc}^{-3}$ and $\mathrm{M}_{\star}(\mathrm{B})=-20.6$. Assuming that half of all galaxies, whatever their luminosity, are surrounded by spherical halos, one gets

$n_{o} \sigma_{o}=\frac{\pi}{2} \Gamma\left(\frac{7}{12}\right) \phi_{o} R_{\star}^{2}$ 
with the above assumptions the Holmberg radius of an $L_{*}$ galaxy (at $\left.z=0\right)$ is $\mathrm{R}_{H}=22 \mathrm{kpc}$.

The size of the absorbers is obtained assuming no cosmological evolution, $\alpha=0$, and an open universe, $\mathrm{q}_{o}=0$. The values derived for $\mathrm{R}_{*}$ are given in Table 1. The dependence on $\mathrm{H}_{c}$ is eliminated by normalizing to $\mathrm{R}_{H}$. The MgII surveys at low $\mathrm{z}$ and the CIV sample were built from blue surveys (Young et al. 1982, Bergeron and Boissé 1984, Tytler et al. 1987). A red survey done by Lanzetta et al. (1987) gives properties of MgII absorption doublets at high $\mathrm{z}$. The Lyman limit sample compiled by Bechtold et al. (1984) refers to absorbers optically thick at their Lyman limit (LLS).

The size of the absorbing gaseous envelopes is a function of the rest equivalent width limit, $\mathrm{w}_{r, l i m}$, of the surveys. This is clearly shown in Table 1 for the MgII systems. There is also an excess of small CIV doublets, $\mathrm{w}_{r}(\mathrm{CIV} \lambda 1548)$ in the range 0.05 to $0.20 \AA$, in the data presented by Meyer and York (1987) and Bergeron and D'Odorico (1987). The radii of the ionized envelopes are at least equal to 4 to $5 \mathrm{R}_{H}$ which is about 3 times larger than the average dimension, $\sim 1.5 \mathrm{R}_{H}$, of $\mathrm{HI}$ discs around spiral galaxies. These discs have sharp edges at $\mathrm{N}(\mathrm{HI}) \sim 10^{19} \mathrm{~cm}^{-2}$ as recently discovered from observations with the VLA (see e.g. Sancisi, 1987).

Table 1. Average size of the absorption line systems

\begin{tabular}{llllll}
\hline system & $\mathrm{w}_{r, \text { lim }}(\AA)$ & $\frac{d N}{d z}$ & $\overline{\mathrm{z}}$ & $R_{\star} / R_{H}$ & references \\
\hline MgII & 0.6 & 0.26 & 0.51 & 2.9 & 1 \\
& 0.25 & 0.69 & 0.5 & 4.7 & 1 \\
& 0.6 & 0.85 & 1.67 & 3.9 & 2 \\
CIV & 0.3 & 1.25 & 1.62 & 4.8 & 2 \\
LLS & 0.3 & 1.64 & 1.70 & 5.4 & 3 \\
\hline
\end{tabular}

references. 1 : Tytler et al. (1987). 2 : Lanzetta et al. (1987). 3 : Bergeron and Boissé (1984). 4 : Bechtold et al. (1984).

Cosmological evolution of absorption line systems is usually expressed in terms of the index $\gamma$

$\frac{d N}{d z} \propto(1+z)^{\gamma}$,

with $\gamma=1$ for $\mathrm{q}_{o}=0$ and $\gamma=0.5$ if $\mathrm{q}_{o}=0.5$.

The redshift evolution of metal systems is difficult to determine since their density is not very high. Smaller uncertainties are obtained when a large redshift interval is sampled, as for the MgII systems. Results are presented in Table 2. The observed samples have been analyzed using a maximum likelihood method. For MgII, Kunth (1987) has combined a new UV survey (Boulade et al. 
1987) to that of Tytler et al. (1987). For CIV, Boissé (1987) has reanalyzed the data compiled by Bergeron and Boissé (1984) and has also added observations by Foltz et al. (1986). A value of $\gamma$ derived from an unpublished survey of Sargent, Boksenberg and Steidel is also quoted.

Table 2. Cosmological evolution of metal systems

\begin{tabular}{lllcc}
\hline ion & $\mathrm{w}_{r, \text { lim }}(\AA)$ & $\mathrm{z}$ range & $\gamma$ & references \\
\hline MgII & 0.6 & $0.2-2.05$ & $2.4 \pm 0.8$ & 1 \\
& & & $2.0 \pm 0.5$ & 2 \\
CIV & 0.3 & $1.4-2.05$ & $1.0 \pm 2.0$ & 3 \\
& & & $1.8 \pm 1.5$ & 3 \\
& 0.15 & $1.4-3.1$ & -1.2 & 4 \\
LLS & & $0.5-3.3$ & $1.3 \pm 1.5$ & 5 \\
\hline
\end{tabular}

references. 1 : Lanzetta et al. (1987). 2 : Kunth (1987). 3 : Boissé (1987). 4 : Sargent (private communication). 5 : Bechtold et al. (1984).

A positive evolution is most probably present ( $2 \sigma$ level) for the MgII systems. Since they should constitute a large fraction of the Lyman limit systems, the different values of $\gamma$ found for the two samples may just reflect the large uncertainties involved. The possible opposite evolution derived from published CIV data and the new larger sample of Sargent et al. may not be only a consequence of uncertainties inherent to small samples. The redshift ranges covered are different. The statistical results presented by Steidel (1987) for $\mathrm{dN} / \mathrm{dz}$ as a function of $\mathrm{z}$ suggest variations in the gradient of $\mathrm{dN} / \mathrm{dz}$; a strong negative evolution could indeed be present at $z>2$ for the CIV systems, as observed for the density of quasars. Results are more ambiguous at lower redshift and to ascertain which type of evolution prevails at $\mathbf{z}<2$ may have to await a CIV UV survey with the Hubble Space Telescope.

\section{IONIZATION LEVEL}

The ionization level of the absorbers is well defined only when low and high ions are observable in the optical thus for $z>1.2$. At these redshifts three phases are present, characterized essentially by the presence/absence of the CIV and MgII doublets. We will mainly outline properties related to the opacity of ionizing photons, multiplicity of the systems and velocity spread of the subcomponents, the velocity of the absorber relative to that of the quasar, and the redshift range thus a possible cosmological evolution. We first restrict the discussion to systems at $\mathrm{z}>1.2$. 


\subsection{Systems of high ionization level $(H)$}

In these systems apart from the $H$ Lyman series, the lines usually detected are the CIV doublet and occasionnally the SiIV doublet. In a few cases NV is present even for $\mathbf{z}_{a}<\mathbf{z}_{e}$ systems. Lines from singly ionized and neutral elements are not detected even in high signal to noise spectra. The ionization level can be defined by the value of the line equivalent width ratio

$$
\frac{\mathrm{CIV}}{\mathrm{MgII}}=\frac{\mathrm{w}_{r}(\mathrm{CIV} \lambda 1548)}{\mathrm{w}_{r}(\operatorname{MgII} \lambda 2796)}
$$

For high ionization level absorbers the lower limit of CIV/MgII can reach 10 to 40 (see e.g. Bergeron and D'Odorico 1986). These systems are also usually weak with $\mathrm{w}_{r}(\operatorname{CIV} \lambda 1548)<1 \AA$.

The opacity to ionizing radiation of the absorbers can be derived by comparing statistical properties of CIV and Lyman limit systems, as done by Bergeron et al. (1987a). From the rate of occurrence of optically thick Lyman limits in their LLS sample, Bechtold et al. (1984) infer that systems with $\mathrm{w}_{r}($ Ly $\alpha)>1.7$ have a discontinuity at the Lyman limit. Although the bulk of high ionization level systems have $\mathrm{w}_{r}(\operatorname{Ly} \alpha)<1.7 \AA$, there are some cases $(\sim 10$ to $20 \%)$ for which $\mathrm{w}_{r}(\operatorname{Ly} \alpha)$ can reach 2 to $3 \AA$. It is yet to be investigated whether the latter show weak lines of $\mathrm{CII}$ and SiII, although MgII or FeII could remain undetected, as it is the case for the optically thick $\mathrm{z}=1.649$ system in PKS0215+015 (Blades et al. 1985, Bergeron and D'Odorico 1986).

The HI density distribution is particularly difficult to determine for the high ionisation level systems. Most often the HI column density, N(HI), cannot be derived from studies of their optically thin Lyman limit, whereas the Ly $\alpha$ line is saturated and on the logarithmic part of the curve of growth. Thus it is usually necessary to estimate the velocity dispersion $b$ from the CIV doublet, assume that the same value of $b$ applies for $\mathrm{HI}$ and CIV, and be sure that individual components have been isolated which requires a spectral resolution of at least FWHM=1 $\AA$ (to resolve 2 components at $\mathrm{z}=2$ spanning $80 \mathrm{~km} \mathrm{~s}^{-1}$ ).

Some high ionization level systems are multiple, but the extreme cases of multiplicity do not belong to this class of absorbers. The velocity spread of the subcomponents is moderate, up to $300 \mathrm{~km} \mathrm{~s}^{-1}$ in some cases, whereas the velocity dispersion of individual components is within the range $b=5$ to $20 \mathrm{~km} \mathrm{~s}^{-1}$. The multiple systems are usually those of larger $\mathrm{w}_{r}(\mathrm{CIV} \lambda 1548)$.

\subsection{Systems of mixed ionization level (M)}

For these absorbers low and high ions are present. The absorption lines are usually strong with $\mathrm{w}_{r}(\operatorname{CIV} \lambda 1548)$ and $/$ or $\mathrm{w}_{r}(\operatorname{Ly} \alpha)>2 \AA$.

The ionization parameter $\mathrm{CIV} / \mathrm{MgII}$ has values within the range 0.3-2.5. Therefore there is a clear discontinuity of CIV/MgII between the high and mixed 
ionization level systems, and a definite tendency for the systems with increasing $\mathrm{w}_{r}(\mathrm{MgII} \lambda 2796)$ to have a decreasing value of the ionization parameter as found by Boissé and Bergeron (1985) and confirmed by Lanzetta et al. (1987: see their Fig.11).

As mentioned above from statistical arguments, most if not all of these absorbers should have optically thick Lyman limits. This is verified when observations of the Lyman limit, in the optical or the UV, are available (Bechtold et al. 1984 ; see also the review of Bergeron et al. 1987a) .

These systems often have a complex velocity structure. Extreme cases show 10 to 15 components spanning up to $2000 \mathrm{~km} \mathrm{~s}^{-1}$. Amongst the subsystems there is often a mixture of high and mixed ionization levels (Pettini et al. 1983, Foltz et al. 1986) and the $M$ systems tend to span a smaller velocity range than the overall complex (Lanzetta et al. 1987). There is however some multiple $M$ systems with all subcomponents of mixed ionization level, as the $\mathrm{z}=1.345$ system in PKS0215+015 (Bergeron and D'Odorico 1986).

The $\mathrm{z}_{a} \sim \mathrm{z}_{e}$ systems, which are typically found in steep-spectrum radioloud quasars, often show multiple structure (Foltz et al. 1986), including extreme cases as the two complexes in GC1556+335 (Morris et al. 1986). This tendency to get an excess of $\mathrm{z}_{a} \sim \mathrm{z}_{e}$ systems in steep-spectrum radio quasars is also present at $\mathrm{z}<1.2$ : the $4 \mathrm{z}_{a} \sim \mathrm{z}_{e}$ systems known at low $\mathrm{z}$ are in radio-loud quasars, 3 being steep-spectrum radio sources (Bergeron et al. 1987a). It is among the $\mathbf{z}_{a} \sim \mathbf{z}_{e}$ systems that the larger spreads of ionization level are found, usually from singly to four times ionized elements, even showing sometimes heavy neutral atoms together with NV as in the $\mathrm{z}=0.401$ system in PKS1912-54 (Bergeron and Boissé 1986). However $\mathrm{z}_{a} \sim \mathrm{z}_{e}$ systems often belong to the $\mathrm{H}$ class.

\subsection{Systems of low ionization level (L)}

These absorbers have been discovered by Lanzetta et al. (1987) in their red survey. Absorption lines of $\mathrm{CII}, \mathrm{MgII}$ and FeII are present but the CIV doublet is undetected. There are only $5 \mathrm{~L}$ systems in this red sample. The absorption lines are fairly strong with $\mathrm{w}_{r}(\mathrm{MgII} \lambda 2796)$ in the range 0.4 to $1.7 \AA$.

The upper limit on the ionization parameter CIV/MgII is 0.5 in 4 cases and 0.2 for the $\mathrm{z}=1.636$ system in PKS0237-233 using the CIV limit given by Boroson et al. (1978). Therefore there is no clear discontinuity between the $\mathrm{M}$ and $\mathrm{L}$ systems contrary to what was found for the $\mathrm{M}$ and $\mathrm{H}$ systems.

About half of the damped Ly $\alpha$ absorbers $\left(\mathrm{N}(\mathrm{HI}) \geq 10^{20} \mathrm{~cm}^{-2}\right)$ contain only metals in a low ionization level (Wolfe 1987), and belong to the L class. Do all $\mathrm{L}$ absorbers are damped Ly $\alpha$ systems ? One of the five systems found by Lanzetta et al. (1987) has a damped Ly $\alpha$ line. No information on $\operatorname{Ly} \alpha$ is available for the remaining four cases, for which this line is shortward of $3300 \AA$. UV observations are needed to specify the link between $\mathrm{L}$ and damped Ly $\alpha$ systems. 
Too few $\mathrm{L}$ absorbers are known and they have not been observed at high enough spectral resolution to conduct a proper study on their multiplicity. However for a few multiple systems of mixed ionization level there are components of low ionisation state.

In summary the stronger systems at $\mathrm{z}>1.2$ are a) of mixed or low ionization level, b) optically thick to ionizing radiation, c) have large column densities and/or multiple components spanning a large velocity range. In our Galaxy only the $\mathrm{M}$ and $\mathrm{L}$ classes have been detected. The percentage of $\mathrm{H}, \mathrm{M}$ and $\mathrm{L}$ systems at $\overline{\mathbf{z}} \sim 1.7$, in the survey of Lanzetta et al. (1987), equals 49,37 and $14 \%$ respectively. Thus contrary to our knowledge prior to this red survey, only about half of the metal-rich absorbers are of high ionization level.

\subsection{Systems at low redshift}

At $\mathrm{z}<1.2$, systems of only mixed or low ionization level are known since no UV absorption line survey is yet possible. Four of these systems, observed with IUE, show a strong discontinuity at the Lyman limit and strong but no damped Ly $\alpha$ lines. However there are two fairly weak MgII systems which do not show any break at the Lyman limit. In one case the system is certain, with saturated MgII lines of moderate strength $(\mathrm{z}=0.852$ in AO $0235+164)$, but the UV data are very noisy (Snidjers et al. 1982). Although UV observations of better $\mathrm{S} / \mathrm{N}$ ratio are needed for confirmation, this suggests that at low redshift some of the weaker systems of low ionizing level are optically thick to ionizing radiation which should imply a cosmological evolution of the ionization state of the absorbers.

There is an excess of weak MgII systems in the samples of Tytler et al. (1987) and Boulade et al. (1987) at $0.25<\mathrm{w}_{r}(\operatorname{MgII} \lambda 2796)<0.6 \AA$, which is not present in the survey of Lanzetta et al. (1987). Although the latter does not go as deep as the lower $z$ surveys, this could also point towards a cosmological evolution of the ionization state of the weaker systems.

Some of the low z MgII systems have an associated CIV absorption as shown by IUE observations of bright quasars (Bergeron et al. 1987a) but the low sensitivity of IUE prevents any conclusion when the CIV doublet is undetected (Bergeron and Kunth 1983). Further low redshift absorbers of high ionization level remain to be discovered.

Complex velocity structure, difficult to reconcile with motions within a single isolated galaxy, are also found in low $\mathrm{z}$ MgII absorbers as for the $\mathrm{z}=\mathbf{0 . 8 5 2 6}$ system in PKS1327-206 with 6 components spanning $560 \mathrm{~km} \mathrm{~s}^{-1}$ (Bergeron et al. $1987 \mathrm{~b}$ : see their Figs. 1 and 2). Multiple systems spanning at most $300 \mathrm{~km} \mathrm{~s}^{-1}$ are also often present in low z MgII absorbers (Bergeron and D'Odorico 1987). 


\section{PREDICTED PROPERTIES OF THE ABSORBING GALAXIES}

The first attempt to detect the absorbing galaxy associated with low z MgII absorbers was made by Weymann et al. (1978), but the sensitivity of the detectors used then was too low to unambiguously find these galaxies. Later another unsuccessfull attempt was made by Carswell et al. (1984) for the $\mathrm{z}=0.356$ and $0.359 \mathrm{MgII}$ absorbers in Q1101-264, although more sensitive detectors were used. Recently a MgII system, $\mathrm{z}=0.430$ in PKS2128-12, was successfully identified (Bergeron 1986).

To understand whether that detection was fortuitous or not, we have estimated the expected average magnitude of an absorbing galaxy and its average angular separation from the sight line to the quasar.

The fraction of galaxies giving rise to $\mathrm{MgII}$ absorption systems brighter than a given luminosity L can be derived from equations (1), (2) and (3)

$$
\frac{d N(x) / d z}{d N(x=0) / d z}=\frac{\int_{x}^{\infty} \sigma d n}{\int_{0}^{\infty} \sigma d n}=\frac{\Gamma(x, 7 / 12)}{\Gamma(7 / 12)}
$$

We find that $50 \%$ of the absorbing galaxies are brighter than $0.3 \mathrm{~L}_{*}(\mathrm{M}(\mathrm{B})=-$ 19.3). For luminosities $\mathrm{L} \geq \mathrm{L}$ 。 this percentage reduces to $19 \%$ and it reaches $72 \%$ for $\mathrm{L} \geq 0.1 \mathrm{~L}_{*}$. Therefore most of the galaxies responsible for MgII absorption in quasar spectra are brighter than the LMC $(x=0.174)$. The apparent magnitude of $0.3 \mathrm{~L}_{*}$ galaxies is given in Table 3 for different redshifts. Since $\mathrm{K}$ correction has not been applied in the determination of the apparent magnitude, the values of $\mathrm{m}$ in Table 3 correctly refer to an effective band width of $\Delta \lambda=(1+\mathrm{z}) \Delta \lambda(\mathrm{B})$ centered on $\lambda_{o b s}(\mathrm{~B})=4400(1+\mathrm{z}) \AA$.

Table 3. Apparent magnitude and size of $0.3 \mathrm{~L}_{\star}$ absorbing galaxies

\begin{tabular}{llll}
\hline $\mathrm{z}$ & $\mathrm{m}$ & $\lambda_{\text {obs }}(\mathrm{B})(\AA)$ & $\theta(\operatorname{arcsec})$ \\
\hline 0.2 & 22.1 & 5280 & 8.6 \\
0.4 & 22.8 & 6160 & 5.4 \\
0.8 & 24.5 & 7920 & 3.8 \\
\hline
\end{tabular}

The expected angular separation between the absorbing galaxy centre and the quasar can be estimated from equation (1), using the value of $R_{*}$ given in Table 1 for $\overline{\mathrm{z}}=0.51 \mathrm{MgII}$ sample with $\mathrm{w}_{r, l i m}=0.6 \AA$. This angular separation, independent of $\mathrm{H}_{o}$, can be written

$\theta_{*}=5.23\left(\frac{d N / d z^{\prime}}{1+z^{\prime}}\right)^{0.5} \frac{(1+z)^{2}}{z+z^{2} / 2} \operatorname{arcsec}$, 
$\theta(x)=\theta_{\star} x^{5 / 12}$

Numerical estimates of $\theta(x=0.3)$ are given in Table 3.

The absorbing galaxies should thus be bright enough and well separated on the sky from the quasr image to be easily detectable with present day technics. Further the galaxy G2128-12, identified with the MgII absorber at $\mathrm{z}=0.430$ towards PKS2128-12, is 8.6 arcsec away on the sky from the quasar and has a magnitude $\mathrm{m}(\mathrm{V})=\mathbf{2 1 . 5}$, properties close to the predicted ones. This led us to think that the negative attempts reported prior to 1985 were due in part to the low sensitivity of the instrumentation used.

\section{THE MGII ABSORBING GALAXY SAMPLE}

Our sample comprises two MgII absorption systems at the quasar emission redshift $\mathrm{z}_{e}$ and 9 at $\mathrm{z}_{a} \ll \mathrm{z}_{e}$. The redshift range covered by the absorbers is $0.16-0.79$ and the rest equivalent width of $\operatorname{MgII} \lambda 2796$ has values between 0.40 and $3.0 \AA$. When this survey started the homogeneous sample of $\mathrm{MgII}$ absorption systems of Tytler et al. (1987) was not yet available, and we selected published cases with data of good $\mathrm{S} / \mathrm{N}$ ratio restricted to resolved MgII doublets. To our knowledge there is no obvious bias in our sample, at least for the $\mathrm{z}<0.5$ absorbers. Searches for $\mathrm{z}>0.5 \mathrm{MgII}$ absorbing galaxies were done only in optimum seeing conditions $(\mathrm{FWHM} \leq 1.3 \mathrm{arcsec})$. All the observations were carried out with the $3.6 \mathrm{~m}$ telescope at the European Southern Observatory (ESO), La Silla, Chile. Since at first we only got positive detections of the absorbing galaxy, we have started observing previous negative cases. Recent searches for the absorbing galaxies done by other researchers to a sensitivity level similar to that of our observations are also included in the sample.

We briefly summarize results for the $\mathrm{z}_{a} \sim \mathrm{z}_{e}$ systems. They provide information on the cluster usually associated with radio-loud quasars. We got observations only of two absorbers G2135-14 at $\mathrm{z}=0.20$ and G1912-54 at $\mathrm{z}=0.401$ (Bergeron and Boissé 1986). Their angular distance to the quasr is smaller by a factor of 8 and 2 respectively than expected from the distance of the nearest neighbour in the quasr sample of yee and Green (1984). Recent data obtained for the galaxy closest on the sky to the quasar PKS1912-54 (angular separation of $8.3 \mathrm{arcsec}$ ) show that its redshift is identical to that of the MgII absorption system. In the galaxy spectrum, there are strong stellar absorption lines of CaII, HI and the $\mathrm{G}$ band and possibly a weak [OII] emission line (Bergeron, unpublished).

The $\mathrm{z}_{a} \ll \mathrm{z}_{e}$ sample comprises 13 systems out of which 10 with a clear identification of the absorbing galaxies. Some properties of the absorbers are given in Table 4. The angular separation between the galaxy centre and the quasar, $\theta$, varies between 0.9 to $3.3 \mathrm{R}_{H}\left(\mathrm{~L}_{*}\right.$ at $\left.\mathrm{z}=0\right)$ with a mean equal to $2.3 \mathrm{R}_{H}$ for the 10 identified galaxies. For two $\mathrm{MgII}$ systems there is a possible candidate for which 
either inconclusive (G1101-26) or no (G1229-02) spectroscopic observations are available. These two possible absorbers have angular separation and magnitude similar to those derived for the identified cases.

All the MgII galaxies are intrinsically bright. This is not a consequence of an observational bias since these absorbers are about 2 magnitudes brighter (or more) than our detection limit $\mathrm{m}(\mathrm{r}) \simeq 24$. Galaxies of the SMC and LMC type could indeed have been detected up to redshifts of 0.21 and 0.50 respectively. The spread in $M(r)$ is only of about 2 magnitudes with an average $M(r)=-21.1$. No $\mathrm{K}$ correction has been applied to derive $\mathrm{M}(\mathrm{r})$. Since the absorbers cover a wide redshift range, it is possible to derive an absolute $B$ magnitude in the galaxy rest frame only for some of the galaxies $(\mathrm{z} \leq 0.5)$. For the overall survey we can derive an intrinsic $\mathrm{M}(\lambda 3700)$ magnitude as done by Bergeron and Boissé (1987) for a sample somewhat larger than presented in this review.

Table 4. Properties of MgII absorbing galaxies

\begin{tabular}{lllcllll}
\hline quasar & & & & & & \\
& $\mathrm{z}_{a}$ & type & $\theta(")$ & $\mathrm{R} / \mathrm{R}_{H}$ & $\mathrm{~m}(\mathrm{r})$ & $\mathrm{M}(\mathrm{r})$ & references \\
\hline & & & & & & & \\
$0151+04$ & 0.160 & em & 6.4 & 1.1 & 19.1 & -20.7 & 1 \\
& & em & 10.9 & 1.9 & 20.2 & -19.6 & 1 \\
$1127-14$ & 0.313 & em & 9.5 & 2.7 & 19.3 & -22.1 & 2 \\
$2128-12$ & 0.430 & em & 8.6 & 2.9 & 21.0 & -21.1 & 3 \\
$1511+10$ & 0.437 & em & 6.7 & 2.4 & 22.0 & -20.3 & 2 \\
$1038+06$ & 0.441 & em & 9.6 & 3.3 & 21.0 & -21.3 & 2 \\
$0109+20$ & 0.535 & abs & 7.0 & 2.7 & 21.7 & -21.0 & 2 \\
$2145+06$ & 0.790 & em & 5.9 & 2.7 & 22.1 & -21.7 & 2 \\
$0952+17$ & 0.238 & no galaxy found at $\mathrm{z}_{a}$ & & & 2 \\
$1229-02$ & $0.395^{a}$ & - & $8.6^{b}$ & 2.6 & 22.3 & $(-19.8)$ & 2 \\
\hline $1332+55$ & 0.373 & abs & 5.0 & 1.6 & $20.7:$ & $-21.3:$ & 4 \\
$1209+07$ & 0.393 & HII & 7.1 & 2.3 & 21.9 & -20.2 & 5 \\
$0235+16$ & $0.524^{a}$ & HII & 2.3 & 0.9 & & & 6 \\
$1101-26$ & 0.356 & - & $11.0^{b}$ & 3.3 & 20.7 & $(-21.2)$ & 7,2 \\
& 0.359 & & & & & &
\end{tabular}

${ }^{a}$ sytems with $21 \mathrm{~cm}$ absorption detected

${ }^{b}$ possible candidates, not yet confirmed spectroscopically

references. 1 : Bergeron et al. (1987c). 2 : this work, 3 : Bergeron (1986), 4 :

Miller et al. (1987), 5 : Cristiani (1987), 6 : Smith et al. (1977), 7 : Carswell et al. (1984). 
The $\mathrm{z}_{a} \ll \mathrm{z}_{e}$ absorbing galaxies are not in dense clusters. For most fields we have at least the second galaxy closest on the sky to the quasar and our largest number of galaxies per field with identified redshfit is five. In only two cases there is a second object at $\mathrm{z} \sim \mathrm{z}_{a}$. Therefore most of the absorbing galaxies are field galaxies and sometimes they belong to loose groups.

A striking property of the galaxies with large gaseous envelopes is their spectral type. They all have blue continuum with $\mathrm{F}(\lambda) \sim$ constant shortwards of $\lambda 3727$, down to $\lambda_{r} 2140$ for the $z=0.79$ absorber. Eight out of the ten galaxies show emission lines, essentially [OII $] \lambda 3727$, among which two resemble giant HII regions with a weak continuum and very strong Balmer emission lines and forbidden [OII] and [OIII] lines (see Fig. 2 in Cristiani 1987). The whole gaseous envelope of these HII galaxies should have detectable extended [OII] emission as observed for G0235+16 (Cohen et al. 1987). The other six emission line galaxies have spectra similar to that of G2128-12 (see Fig. 3 in Bergeron 1986) with both emission lines and resolved stellar absorption lines. Their $[\mathrm{OII}] \lambda 3727$ rest equivalent width ranges from 15 to $30 \AA$ whereas that of HII galaxies reaches about $100 \AA$. Thus all the galaxies with very extended gaseous envelopes identified so far show signs of recent or present strong stellar formation activity. The percentage of field galaxies at $\mathrm{z} \sim 0.5$ with colors and spectra similar to those of the absorbing galaxies is not accurately known. Ellis (1987) gives a fraction of $20-30 \%$ at $\mathrm{z} \sim 0.3$ for galaxies with unusually strong $[\mathrm{OII}], \mathrm{w}_{r}([\mathrm{OII}] \lambda 3727)>40 \AA$, which is comparable to the number given for cluster at similar redshift. From his multicolor photometric survey Koo (1986) suggests that the fraction of field galaxies at $\mathrm{z} \sim 0.4$ intrinsically bluer than $\mathrm{B}-\mathrm{V}=0.7$ is $\sim 74 \%$ while the local value is $\sim 40 \%$. The galaxies of our sample are at least as blue as G2128-12 for which we got $\mathrm{B}-\mathrm{V}=0.5$.

If we consider the number given by Ellis we should multiply the values for $R_{\star}$ given in Table 1 by roughly $\sqrt{2}$ which would imply halos sizes larger than actually observed. Therefore our survey suggests that a large fraction $(\sim 50 \%$ or more if our adopted value of $\phi_{o}$ is an overestimate : see e.g. De Lapparent et al. 1986) of the field galaxies have properties similar to those observed for galaxies with very extended halos. To clarify this problem would require large spectroscopic surveys of field galaxies as those, unpublished, of Dressler, Ellis and Koo. Our test sample of field galaxies, which are not identified with the absorber, will also be of some value.

Extended gaseous envelopes associated with nearby galaxies have been detected by their CaII absorption in quasar spectra. The first discovery was made by Boksenberg and Sargent (1978) and there is now 5 identified cases presented in Table 5. The angular separation between the galaxy centre and the quasar ranges between 0.7 and $1.3 \mathrm{R}_{H}$ whereas for the MgII galaxies it could reach $3.3 R_{H}$. The large CaII galaxy-quasar pair survey done recently by Morton et al. (1986) has 
Table 5. Properties of the CaII absorbing galaxies

\begin{tabular}{lllllll}
\hline quasar & $\mathrm{z}_{a}$ & $\mathrm{R} / \mathrm{R}_{H}$ & $\begin{array}{l}\mathrm{w}_{r}(\mathrm{CaII} \mathrm{K}) \\
(\AA)\end{array}$ & $\begin{array}{c}\mathrm{w}_{r}(\mathrm{MgII} 2796) \\
(\AA)\end{array}$ & $\begin{array}{l}\mathrm{N}(\mathrm{HI}) \\
\left(\mathrm{cm}^{-2}\right)\end{array}$ & references \\
\hline $0955+32$ & 0.0047 & 0.75 & 0.43 & $6.5(\mathrm{D})$ & $21 \mathrm{~cm} \mathrm{abs}$ & 1,2 \\
$1327-20$ & 0.0180 & 0.91 & 0.49 & & $21 \mathrm{~cm} \mathrm{abs}$ & 2,3 \\
$0154+04$ & 0.0188 & 1.27 & 0.57 & & & 4 \\
$2020-37$ & 0.0287 & 0.77 & 0.35 & & $21 \mathrm{~cm} \mathrm{abs}$ & 5,3 \\
$0446-21$ & 0.0668 & 1.05 & 0.96 & & & 6 \\
$2128-12$ & 0.4299 & 2.9 & 0.11 & 0.40 & $210^{17}-810^{18}$ & 7,2 \\
$0454-22$ & 0.4744 & - & 0.13 & 1.37 & & 8 \\
$0002-42$ & 0.8366 & $3.6^{a}$ & 0.60 & 4.68 & & 9,2 \\
\hline
\end{tabular}

D refers to both lines of the doublet

${ }^{a}$ possible candidates, not yet confirmed spectroscopically

references. 1 : Boksenberg and Sargent (1978). 2 : Bergeron et al. (1987b). 3 : Boissé et al. (1987). 4 : Bergeron et al. (1987c). 5 : Boksenberg et al. (1980). 6 : Blades et al. (1981). 7 : Bergeron (1986). 8 : Robertson et al. (1987). 9 : Bergeron and Boissé (1987).

not revealed any new CaII envelopes. For most of their cases the angular separation is unfortunately larger than $3.5 \mathrm{R}_{H}$, distance at which we expect very few detection of gaseous envelopes from the results of our MgII galaxy survey.

For 3 out of the $4 \mathrm{CaII}$ absorption systems at low $\mathrm{z}$ detected in spectra of radio-loud quasars there is an associated $21 \mathrm{~cm}$ absorption (Table 5). The small sizes of these CaII envelopes are consistent with those usually observed for HI discs, which have been found recently to have sharp edges around $1.5 \mathrm{R}_{H}$ (see e.g. Sancisi 1987). The identified MgII galaxy with detected $21 \mathrm{~cm}$ absorption (G0235+16 in Table 4) is also of small extent.

Ca II absorption from MgII envelopes has been detected in a few cases reported in Table 5. They are weaker than observed for the CaII galaxies, except for a very strong MgII system. Further for one of the low redshift CaII galaxies a strong MgII absorption has been detected in the UV. The results presented in Table 5 may suggest a correlation between the radial distance to the galaxy centre and the absorption line equivalent width (or ionic column density). However there is only a weak trend of increasing projected separation with decreasing MgII rest equivalent width for the sample of identified MgII absorbers (Bergeron and Boissé 1987). Column densities should be used instead of $\mathrm{w}_{r}$ but they cannot always be determined from available data. Other parameters such as the galaxy luminosity (scaling R with $\mathrm{L}$ ) should also be considered. 
The magnitude $\mathrm{M}(\mathrm{r})$ of the galaxies with large $\mathrm{MgII}$ envelopes are within the range -20.3 to -22.1 with $<M(r)>=-21.1$. Including the two additional possible candidates increases only slightly this range towards the faint end to $\mathbf{- 1 9 . 8}$ and does not modify the value of $\langle\mathrm{M}(\mathrm{r})\rangle$. The average redshift of the galaxy sample equals 0.43 with an rms of 0.16 . Thus the absolute magnitudes $M(r)$ given in Table 4 are for most MgII galaxies fairly close to an intrinsic B magnitude, and the majority of absorbing galaxies are brighter than $\mathrm{L}_{*}$.

Our success rate of the absorber identification is $77 \%$ and increases to $92 \%$ when including the possible candidates. As already mentioned, absorbers with luminosity as that of the LMC could have been detected up to $z=0.50$. Our results appear inconsistent with a $\mathrm{MgII}$ galaxy luminosity function without cut-off towards the faint end since a detection rate of about $80 \%$ and no cut-off would imply that half of the identified absorbers be fainter than $M(r)=-19.8$ whereas none was so.

To determine more accurately the expected success rate one should not only consider a cut-off in the luminosity function at $x_{c}=\mathrm{L}_{c} / \mathrm{L}_{*}$ but also the effect of the seeing which prevents detecting galaxies too close on the sky to the quasar. The latter implies that galaxies of small size, thus intrinsically faint, cannot be identified from ground-based observations. However if we assume that objects closer on the sky than $\theta_{s}=2$ arcsec from the quasar are not detectable, only very faint galaxies $x<0.03$ are unobservable at $\mathrm{z}=0.4$. The probability to identify the absorber with the above assumptions is

$$
P=\left[\Gamma\left(7 / 12, x_{c}\right)-x_{s}^{5 / 6} \Gamma\left(-1 / 4, x_{c}\right)\right] / \Gamma\left(7 / 12, x_{c}\right),
$$

where $x_{s}$ is the normalized luminosity of a galaxy with a MgII envelope of radius $\boldsymbol{\theta}_{s}$. Through $x_{s} \mathrm{P}$ is a function of $\mathrm{z}$. To reach a success rate of about $80 \%$ at $\mathrm{z}=0.4$ and 0.8 implies a cut-off at $x_{c}=0.1$ and 0.3 respectively. This is consistent with the faintest magnitude observed $\mathrm{M}(\mathrm{r})=-19.8$ or $x=0.48$. Introducing a detectability limit, $x_{V}>x_{c}$, decreases further $\mathrm{P}$ which is still given by eq. (9) but with $x_{c}$ replaced by $x_{V}$ in the numerator.

A cut-off in the luminosity function should be taken into account when estimating the MgII galaxy radius. Values of $\mathrm{R}_{\star}$ given in Table 1 should be multiplied by $\left[\Gamma(7 / 12) / \Gamma\left(x_{c}, 7 / 12\right)\right]^{0.5}$ which equals $\sqrt{2}$ for $x=0.3$.

Another way to reconcile our observations with a luminosity function without cut-off would be to invoke a cosmological evolution of the galaxy luminosity. However this assumption is not supported by the galaxy surveys at $\mathrm{z} \sim 0.4$ of Koo (1986) and Ellis (1987).

It is unlikely that a faint galaxy right on the sight line to the quasar be responsible for the absorption system while the identified galaxy would only be its nearest neighbour. Both objects should have a redshift equal to $\mathbf{z}_{a}$ within $\sim 200 \mathrm{~km} \mathrm{~s}^{-1}$, i.e. be correlated in position and velocity space which would suggest that bright galaxies are always surrounded by faint satellite galaxies at a radial 
distance $\mathrm{R} \sim 3 \mathrm{R}_{H}$. We can consider the sight line to quasars with absorption systems as a random point in the sky to estimate the expected angular separation between a faint undetected galaxy and a brighter one. Although in our sample the absorber and the quasar have unrelated redshifts, we can use results of the survey by Yee and Green (1984) for galaxies either possibly spatially correlated with quasars or in test fields. The former give, for radio-loud quasars, the density of galaxies in clusters and the latter the density of field galaxies. Their magnitude limit is roughly $\mathrm{m}(\mathrm{Gunn} \mathrm{r}) \sim 21$. Galaxies we identified as the absorbers at $\mathrm{z} \sim 0.4$ would thus have been detectable in their survey but not LMC type galaxies. The average angular distance to the nearest bright neighbour is 18.4 arcsec in a cluster at $\mathrm{z}=0.30$ to 0.45 and $\sim 31.5$ arcsec for field galaxies (as determined for the radioquiet quasar sample and the two test field surveys). For the same redshift range the average angular separation between the absorbing galaxies of our sample and an hypothetical faint galaxy on the sight line to the quasar is 7.0 arcsec, value incompatible with those derived by Yee and Green (1984).

In conclusion, galaxies giving rise to $\mathrm{MgII}$ absorption line systems in quasar spectra have (i) emission spectra with strong emission lines and/or a blue continuum which suggests on-going or recent stellar formation activity, (ii) large gaseous envelopes $\mathrm{R}(\mathrm{MgII}) \sim 2.5 \mathrm{R}_{H}$ (in the assumption of spherical halos), (iii) they are bright $M(r) \sim-21$ (for $\mathrm{z} \sim 0.4$ this is roughly an intrinsic $B$ magnitude) (iv) the contribution of dwarf galaxies to absorption line systems is most probably negligible except if they always cluster around bright ones at a radial distance of $\sim 2.5 \mathrm{R}_{H}$.

There are two main questions which remain open. The first concerns the existence of bright field galaxies without large gaseous envelopes, problem possibly linked to the fraction of field galaxies without strong stellar formation activity. It will require a survey of high $\mathrm{z}$ galaxy-quasar pairs followed by a search for $\mathrm{MgII}$ absorption in the quasar spectra. The second is related to the range in ionization level of the absorbers, thus the existence of gaseous halos of very high ionization state, which calls for a low redshift CIV absorption line survey with the Hubble Space Telescope.

\section{REFERENCES}

Bahcall, J.N. \& Spitzer, L. (1969), Ap. J. Letters 156, L63

Bechtold, J., Green, R.F., Weymann, R.J., Schmidt, M., Estabrook, F.B., Sherman, R.D., Wahlquist, H.D. \& Heckman, T.M. (1984), Ap. J. 281, 76.

Bergeron, J. (1986). Astron. Ap. Letters 155, L8.

Bergeron, J. \& Kunth, D. (1983). Mon. Not. R. Astr. Soc. 205, 1053.

Bergeron, J. \& Boissé, P. (1984). Astron. Ap. 133, 374.

Bergeron, J. \& Boissé, P. (1986). Astron. Ap. 168, 6.

Bergeron, J. \& D'Odorico, S. (1986). Mon. Not. R. Astr. Soc. 220, 833. 
Bergeron, J., Savage, B. \& Green, R.F. (1987a). In The Scientific Accomplishments of the IUE. ed. Y. Kondon. Reidel, Dordrecht, p. 703.

Bergeron, J., D’Odorico, S. \& Kunth, D. (1987b). Astron. Ap. 180, 1.

Bergeron, J., Boulade, O., Kunth, D., Tytler, D., Boksenberg, A. \& Vigroux, L. $(1987 \mathrm{c})$. Astron. Ap., in press.

Bergeron, J. \& D'Odorico, S. (1987). in preparation.

Bergeron, J. \& Boissé, P. (1987), in preparation.

Blades, J.C., Hunstead, R.W. \& Murdoch, H.S. (1981). Mon. Not. R. Astr. Soc. $194,669$.

Blades, J.C., Hunstead, R.W., Murdoch, H.S. \& Pettini, M. (1985). Ap. J. 288, 580.

Boissé, P. \& Bergeron, J. (1985). Astron. Ap. 145, 59.

Boissé, P. (1987). In High Redshift and Primeval Galaxies. Eds J. Bergeron, D. Kunth \& B. Rocca-Volmerange. Editions Frontières, in press.

Boissé, P., Dickey, J.M., Kazès, I. \& Bergeron, J. (1987). Astron. Astrophys., in press.

Boksenberg, A. \& Sargent, W.L.W. (1978). Ap. J. 220, 42.

Boksenberg, A., Danziger, I.J., Fosbury, R.A.E. \& Goss W.M. (1980). Ap. J. Letters 242, L145

Boroson, T., Sargent, W.L.W., Boksenberg, A. \& Carswell, R.F. (1978). Ap. J. $220,772$.

Boulade, O., Kunth, D. \& Tytler, D. (1987). in preparation.

Carswell, R.F., Morton, D.C., Smith, M.G., Stockton, A.N., Turnshek, D.A. \& Weymann, R.J. (1984). Ap. J. 278, 486.

Cohen, R.D., Smith, H.E., Junkkarinen, V.T. \& Burbidge, E.M. (1987). Ap. J. 318,577

Cristiani, S. (1987). Astron. Ap. Letters 175, L1.

De Lapparent, V., Geller, M.J., Huchra, J.P. (1986). Ap. J. Letters 302, L1.

Ellis, R. (1987). In High Redshift and Primeval Galaxies. Eds J. Bergeron, D. Kunth \& B. Rocca-Volmerange, Editions Frontières, in press.

Foltz, C.B., Weymann, R.J., Peterson, B.M., Sun, L., Malkan, M.A. \& Chaffee, Jr.-,F.H. (1986). Ap. J. 307, 504.

Koo, D.C. (1986). Ap. J. 311, 651.

Kunth, D. (1987). In High Redshift and Primeval Galaxies. Eds J. Bergeron, D. Kunth \& B. Rocca-Volmerange. Editions Frontières. in press.

Lanzetta, K.M., Turnshek, D.A. \& Wolfe A.M. (1987). Ap. J., 322, 739.

Meyer, D.M. \& York, D.G. (1987). Ap. J. Letters 315, L5.

Miller, J.S., Goodrich, R.W. \& Stephens, S.A. (1987). Astron. J., 94, 633.

Morris, S.L., Foltz, C.B., Weymann, R.J., Schectman, S., Price, C., Boroson, T.A. \& Turnshek, D.A. (1986). Ap. J. 310, 40.

Morton, D.C., York, D.G. \& Jenkins, D.B. (1986). Ap. J. 310, 583.

Pettini, M., Hunstead, R.W., Murdoch, H.S. \& Blades, J.C. (1983). Ap. J. 273, 436. 
Robertson, J.G., Morton, D.C., Blades, J.C., York, D.G. \& Meyer D.M. (1987). Ap. J., in press.

Sancisi, R. (1987). In QSO Absorption Lines : Probing the Universe. Eds C. Blades, C. Norman \& D. Turnshek, Cambridge University Press, in Press.

Sargent, W.L.W. (1987). IAU Symp. $\mathrm{N}^{0} 124$. Observational Cosmology. Eds A. Hewitt et al., p. 777

Steidel, C.C. (1987). In High Redshift and Primeval Galaxies. Eds J. Bergeron, D. Kunth, B. Rocca-Volmerange, Editions Frontières, in press.

Smith, H.E., Burbidge, E.M. \& Sunkkarinen, V.T. (1977). Ap. J. $218,611$.

Snijders, M.A.J., Boksenberg, A., Penston, M.V. \& Sargent, W.L.W. (1982). Mon. Notr. R. Astr. Soc. 201, 801.

Tytler, D., Boksenberg, A., Sargent, W.L.W., Young, P. \& Kunth, D. (1987). Ap. J., Suppl. 64, 667.

Wagoner, R. (1967). Ap. J. 149, 465

Weymann, R.J., Boroson, T.A., Peterson, B.M. \& Butcher, H.R. (1978). Ap. J. $226,603$.

Weymann, R.J., Williams, R.E., Peterson, B.M. \& Turnshek, D.A. (1979). Ap. J. $234,33$.

Wolfe, A.M. (1987). In QSO Absorption Line : Probing the Universe. Eds C. Blades, C. Norman \& D. Turnshek, Cambridge University Press, in press.

Yee, H.K.C. \& Green, R.F. (1984). Ap. J. $280,79$.

Young, P., Sargent, W.L.W. \& Boksenberg, A. (1982). Ap. J. Suppl. 48, 455. 